\title{
Is Bilingual Education Sustainable?
}

\author{
Francesca Costa and Maria Teresa Guasti *(D) \\ Department of Psychology, University of Milano-Bicocca, 20126 Milan, Italy; f.costa9@campus.unimib.it \\ * Correspondence: mariateresa.guasti@unimib.it
}

Citation: Costa, F.; Guasti, M.T. Is Bilingual Education Sustainable? Sustainability 2021, 13, 13766. https://doi.org/10.3390/ su132413766

Academic Editor: Grigorios

L. Kyriakopoulos

Received: 18 October 2021

Accepted: 6 December 2021

Published: 13 December 2021

Publisher's Note: MDPI stays neutral with regard to jurisdictional claims in published maps and institutional affiliations.

Copyright: (c) 2021 by the authors. Licensee MDPI, Basel, Switzerland. This article is an open access article distributed under the terms and conditions of the Creative Commons Attribution (CC BY) license (https:/ / creativecommons.org/licenses/by/ $4.0 /)$.

\begin{abstract}
We present cross-sectional research to verify whether learning to read in Italian (the participants' mother language and majority language) is delayed when simultaneously learning to read in English (a second language not spoken in the country). Available evidence considering the specific combination of bilingual orthographies being acquired suggests that there should not be adverse effects on the Italian literacy outcomes of Italian-English immersion students. To verify this hypothesis, the Italian reading performance of three groups of bilinguals educated in 50:50 ItalianEnglish immersion programs in Grades 1, 3 and 5 were compared to that of three control groups of Italian monolingual peers attending mainstream monolingual Italian schools. The second aim was to examine the impact of an Italian-English immersion program on English language and literacy skills. To pursue this goal, we examined the English performance of the bilingual group across Grades 1, 3 , and 5. Finally, we aimed to verify whether the language and reading attainments exhibited by the bilingual children in Italian were correlated to their English performance. The results show that bilingual children were not less proficient in Italian than monolingual children; improvement in English was observed across all grades, and performance in Italian was correlated with performance in English.
\end{abstract}

Keywords: bilingualism; biliteracy; immersion education; Italian

\section{Introduction}

Is multilingualism sustainable? Would it not be better if humanity could only speak one language perfectly? The answer to the first question is yes, and that to the second is no. If someone is not convinced of these answers, we suggest reading chapter 11 of Jared Diamond [1], "The world until yesterday". Among other things, Diamond points out that humanity has always been multilingual, and even today, there are areas of the world where people currently speak a mean of five languages. He also notes that "Sadly, languages are now vanishing more rapidly than at any previous time in human history. If current trends continue, $95 \%$ of the languages handed down to us from the tens of thousands of years of history of behaviourally modern humans will be extinct or moribund by the year 2100". As plant and animal species are becoming extinct, so are languages, and both losses are catastrophic. Then, he continues, "Much less attention has been paid to the disappearance of our languages, and their essential role in the survival of those indigenous cultures. Each language is the vehicle for a unique way of thinking and talking, unique literature, and a unique view of the world. Hence looming over us today is the tragedy of the impending loss of most of our cultural heritage, linked with the loss of most of our languages". Losing a language means losing culture and all the practices that this culture has created (the names of plants, ways of building some things and so on).

In light of these considerations, we think that promoting multilingualism is a must. This goal can be achieved in several ways and with different languages. In this article, we present some data showing that multilingualism does not hinder literacy achievement and thus, some negative biases often raised against providing double literacy have no scientific basis. The specific case we are concerned with is becoming bilingual through immersion education. Despite the negative bias toward bilingualism, many families worldwide wish 
for their children to become fluent in English to navigate the global economy in their working future. Thus, the number of schools that offer bilingual education programs (the mother tongue or country language and English) has increased, intending to promote a real bilingual competence and cultural enrichment over time. Rather than merely teaching English as a foreign language, bilingual academic curricula use English as a medium of instruction for school subjects [2]. Among dual-language approaches to bilingual education, in immersion programs, topics are conveyed in both the majority language of the country (the mother tongue or L1, i.e., Italian in Italy) and an additional language (a second language or L2, mainly English in Italy). The age at which children start the immersion experience and the amount of time spent in L2 immersion may vary depending on when the child enters the bilingual education system and the type of immersion program the school pursues.

A child could enter an immersion program very early, from nursery, for example, or from kindergarten. Children are effectively immersed in two languages, thus acquiring a second language implicitly through input during routines, daily activities, songs, and the learning of subject content. When facing literacy acquisition at primary school, children learn to read and write in two languages. The separation of languages exists, and teachers typically present content only in L1 or L2; the two languages are rarely used within the same lesson and are not integrated during instructional time.

As to the specific method used, the two main types of immersion are the 50:50, or simultaneous, and the $90: 10$, or sequential, immersion program. The 50:50, or simultaneous, program is the most widespread immersion program worldwide. It ensures that children are equally exposed to two languages within the same developmental and educational time and growth window $[3,4]$, such that children consistently receive approximately equal amounts of instruction in both languages across all subjects, every day. The instructional day is equally divided between two languages, and subjects are taught $50 \%$ in one language and $50 \%$ in the other.

On the other hand, in 90:10, or sequential, immersion programs, literacy starts to a greater extent in a minority language (which is typically their heritage language), with a minor quantity of instruction in the majority language (i.e., the language spoken in the country where they live). However, instruction in the majority language slowly increases throughout the school years. More precisely, students placed in a sequential immersion program start spending approximately $90 \%$ of the instructional day learning the minority language and the remaining $10 \%$ being instructed in the majority language. Then, this ratio of language instruction rebalances throughout primary school, with minority language immersion gradually decreasing and the use of majority language increasing, usually reaching $50: 50$ by Grade 5, around ages 10-11. In this type of immersion program, students typically start learning how to read and write in the heritage language, their L1, and, subsequently, in the majority language, which is the L2 for them. The 90:10 immersion programs mostly make sense in those countries where students from immigrant families constitute a substantial part of the population in the demographic rate (such as people of Hispanic or Mexican origin in California and Texas, for example). For these students, their heritage language, or L1, could represent a solid base for literacy development in English, and knowledge and skills acquired in the L1 can transfer to the L2 [5,6]. Berens et al. [7] compared the effectiveness of 50:50 and 90:10 immersion programs on the language and reading performance of two groups of Spanish-English second and third graders (ages 7-9), who had been exposed to both Spanish and English since birth or kindergarten (i.e., from three years of age). Their results suggested that a 90:10 immersion program promotes phonological awareness in Spanish and English, but 90:10 learners lag behind 50:50 learners with respect to deeper lexical competence, which may likely be gained in the upper grades. In addition, Berens et al. [7] proposed that the specific combination of the orthographic systems of the L1 and the language to be learned should be taken into consideration when determining which immersion program would be more effective. In particular, the 50:50 immersion program—with training in phonological decoding, manipulation, 
and phoneme-grapheme patterns during the early school years in both languages-may ultimately prove the optimal choice for learning so that children have a solid foundation in both surface and in-depth grammatical features from the beginning of their education. However, Berens and colleagues hypothesized that this phonological reinforcement would be particularly beneficial for children who are native speakers of languages with a deep orthography (e.g., English).

The overarching goal of bilingual education is to learn reading and gain language skills in both languages with high levels of mastery [7], reaching grade-level academic achievements and multicultural competence [8]. From a purely social-cultural perspective, dual-language learning should represent an advantage over traditional single-language education due to the globalization of communication and economy. However, the question of whether bilingual reading exposure may afford reading advantages or disadvantages, compared to traditional monolingual literacy, prevailed in numerous studies.

A conspicuous number of studies found bilingual students gained the same literacy skills as those of their monolingual peers (e.g., [9-12]). Others found that children benefitted from bilingual programs compared to monolingual programs (e.g., [3,5,13-17]). Slavin and Cheung [4] conducted a systematic review of experimental studies focusing on reading attainment in bilingual primary students with different L1s or heritage languages (primarily Spanish). The studies compared biliteracy programs and English-only reading programs for English language learners (ELLs). The majority of the studies found that bilingual approaches led to better English reading measures than monolingual approaches. The remaining studies found no differences. The authors concluded that existing evidence favors children's exposure to bilingual literacy instead of fully English programs, especially bilingual strategies that teach reading in the native language and English at different times each day (see also $[16,18])$.

When it comes to the positive effects of bilingual education on reading achievement, it is pivotal to consider the Age of first oral Exposure (AoE) to the L2. For instance, Baker (2011) [2] found that students in an early immersion program, starting at least at kindergarten, could reach the same monolingual peers' competence in L1 after about five years of schooling. Immersion program students could initially lag behind their monolingual peers - and this can be expected because, in the first few years of the immersion program, instruction is focused on L2 learning-but they usually caught up with their monolingual peers in L1 competence by the end of primary school. Moreover, early on, L2 oral language proficiency has been found to relate to academic success up to Grade 8 [19].). Kovelman, Baker, and Petitto [3] established that the age of first exposure to the L2 impacts the reading development of second-and third-graders (aged 7-9 years). In their study, students from Spanish-speaking homes attending 50:50 Spanish-English bilingual schools were recruited. The age at which they were exposed to English for the first time varied: one group had been exposed to English within the first three years of age (early bilinguals) and a second group between the ages of 3 and 6 (late bilinguals). Finally, they recruited a group of English-only-speaking children who attended mainstream English education and had never been exposed to Spanish, which served as a control group. The results showed that in both languages, early Spanish-English bilingual children performed better than SpanishEnglish children exposed to the L2 after the age of 3. In particular, early bilinguals reached a high level of English reading proficiency, obtaining similar reading scores to their English monolingual peers; they also exhibited a reading performance in Spanish equal to that of the Spanish-speaking children. In contrast, bilinguals who had been exposed to the second language (English) after the age of three lagged behind their English-speaking monolingual peers in reading ability. These findings are consistent with several other studies that have reached similar results, suggesting that an early oral exposure to L2-starting within three years of age-allows for the best academic outcomes (e.g., [20,21]). It can be concluded that the age of first oral bilingual exposure plays a crucial role in language development and has a substantial impact on literacy development. 
In summary, the available scientific evidence suggests that bilingual education and biliteracy do not negatively impact children's literacy achievements. At the same time, it seems that the sooner the bilingual education starts, the better it is for academic achievements. In light of these considerations, in this study, we examined another bilingual situation: Italian-speaking children living in Italy and with Italian as their mother language, attending an Italian-English immersion school. The article is structured as follows. First, we present the rationale of our study and the hypothesis, methods and procedure; then, we report the results, discuss them and offer a conclusion.

\section{The Present Study}

Rationale and Hypothesis

The present study is cross-sectional research, which tried to verify whether becoming literate in English, a language not spoken in the country, negatively impacts literacy achievements in the native language (Italian) when exposure to English started at the age of 3 years; in addition, we aimed to investigate the development in the second language and the relation of it to the native language.

Based on the literature review, we hypothesized that:

1. There should not be significant disadvantages in Italian reading attainments in the bilingual group with respect to the monolingual one;

2. Across grades, children should improve their English language and literacy achievements;

3. Correlations between the language and reading skills in Italian and English should be observed, suggesting that there is a positive transfer from Italian to English;

4. Language abilities in one language are expected to correlate with reading abilities in the same language, supporting the idea that oral language supports reading development.

\section{Materials and Methods}

\subsection{Participants}

Participants were recruited from two private Italian-English bilingual primary schools and three Italian monolingual primary schools in Milan and Rome. In one of the bilingual schools, for first graders, $75 \%$ of the schooling time consists of English immersion, while the remaining $25 \%$ is taught in Italian; subsequently, from Grade 2 to Grade 5, students attend a simultaneous immersion program, with the same amount of exposure to English and Italian, that is 50:50. In the other bilingual school, children attend a 50:50 immersion Italian-English program from Grade 1. Thus, they are exposed to English half of the time and Italian for the other half. In both schools, subjects are taught in both languages. The 50:50 immersion programs were privileged as they seemed to deliver the most effective literacy outcomes.

Participants, both bilingual and monolingual, came from Italian families with an estimated medium-high SES. Due to the failure to get all of the questionnaires filled out by the parents, this limitation was overcome through an accurate approximation. SES was thus evaluated by considering and combining three variables: (1) family income, which was linked to the possession of financial means to enroll a child in a private school (bilingual group); (2) geographical living area: the enlisted primary schools, both bilingual and monolingual, are located in areas where the standards of living are known to be high; (3) level of education, measured with a short questionnaire to be filled out by parents (on a scale from 1 to 5 , where five corresponds to a university degree or a higher level of education). SES homogeneity is an essential point to be considered because some studies investigating differences in performance between monolingual and bilingual groups have failed to control for socioeconomic background adequately (for instance, studies comparing Canadian English monolinguals and Canadian immigrant bilinguals as [22-24]).

Inclusion criteria for bilingual participants were having Italian as their mother tongue and having been exposed to English within the first three years of their life, which means at least since formal kindergarten in Italy. Considering the limit of three as the age of first exposure to English, bilingual students could be all considered as early bilinguals, 
according to Paradis, Genesee and Crago (2011) [25]. As has been previously argued, early bilinguals seem to reach better literacy outcomes in L2 than late bilinguals, which are usually exposed after three to four years of age $[3,26]$. To summarize: only early bilingual pupils were included in the study; both bilingual and monolingual children came from families with a medium-high SES to ensure homogeneity within and between the experimental and control groups. Both bilinguals and monolinguals diagnosed with a Developmental Language Disorder or a Learning Disability or with any special needs were excluded from the sample. The final sample comprised a total of 192 children.

The bilingual group consisted of 121 children attending Grade $1(\mathrm{~N}=48)$, Grade 3 $(\mathrm{N}=43)$ and Grade $5(\mathrm{~N}=30)$, whereas the Italian monolingual group included 71 children attending Grade $1(\mathrm{~N}=21)$, Grade $3(\mathrm{~N}=34)$ and Grade $5(\mathrm{~N}=16)$ (The Italian primary school system consists of 5 grades, with children placed in first grade at the age of 6 years). Considering Grades, the final sample comprised 69 first graders, 77 third graders, and 46 fifth graders. As could be noted, the sample is slightly unbalanced in the three classes. First graders' mean age was 83.8 months $(\mathrm{SD}=4.7)$, third graders' mean age was 106.4 months $(\mathrm{SD}=4.8)$ and fifth graders' mean age was $128.6(\mathrm{SD}=4.9)$, calculated by the total number of subjects. The mean age of the monolingual group was $101.7(\mathrm{SD}=19)$, and the mean age of the bilingual children was $106.8(\mathrm{SD}=15.5)$, calculated in months. The mean ages of the two groups did not differ $(\mathrm{t}(190)=1.9, \mathrm{p}=0.07$. However, looking at individual classes, the bilingual and monolingual subgroups in Grades 1 and 3 differed significantly according to their age. Monolingual first graders $(\mathrm{M}=87.3, \mathrm{SD}=4.1)$ were observed to be significantly older $(\mathrm{t}(67)=4.74, p<0.001)$ than bilingual first graders $(\mathrm{M}=82.3, \mathrm{SD}=4)$, and monolingual third graders $(\mathrm{M}=108.8, \mathrm{SD}=4.7)$ were found to be significantly older $(\mathrm{t}(75)=4.13, p<0.001)$ than bilingual third graders $(\mathrm{M}=104.6$, $\mathrm{SD}=4.1$ ). As we explain in detail in the procedure, they were tested in different periods due to organizational reasons.

The sample was balanced for sex, with 105 participants (54.4\% of the sample) being female and 88 being male (45.6\%). More specific details regarding the subgroups can be found in Table 1.

Table 1. The number of subjects, the average ages in months, and the number of females and males of the following subgroups: bilingual first graders, bilingual third graders, bilingual fifth graders, monolingual first graders, monolingual third graders and monolingual fifth graders.

\begin{tabular}{ccc}
\hline Grades & Bilinguals $(\mathbf{N}=\mathbf{1 2 1})$ & Monolinguals $(\mathbf{N}=\mathbf{7 1})$ \\
\hline Grade 1 & $\mathrm{~N}=48$ & $\mathrm{~N}=21$ \\
\hline Mean age in months & $\mathrm{M}=82.3(\mathrm{SD}=4)$ & $\mathrm{M}=87.3(\mathrm{SD}=4.1)$ \\
\hline Females & $\mathrm{N}=23$ & $\mathrm{~N}=12$ \\
\hline Males & $\mathrm{N}=25$ & $\mathrm{~N}=9$ \\
\hline Grade 3 & $\mathrm{~N}=43$ & $\mathrm{~N}=34$ \\
\hline Mean age in months & $\mathrm{M}=104.6(\mathrm{SD}=4.1)$ & $\mathrm{M}=108.8(\mathrm{SD}=4.7)$ \\
\hline Females & $\mathrm{N}=25$ & $\mathrm{~N}=19$ \\
\hline Males & $\mathrm{N}=18$ & $\mathrm{~N}=15$ \\
\hline Grade 5 & $\mathrm{~N}=30$ & $\mathrm{M}=128.2(\mathrm{SD}=4.1)$ \\
\hline Mean age in months & $\mathrm{M}=128.8(\mathrm{SD}=5.3)$ & $\mathrm{N}=8$ \\
\hline Females & $\mathrm{N}=18$ & $\mathrm{~N}=8$ \\
\hline Males & $\mathrm{N}=13$ &
\end{tabular}

\subsection{Materials}

Children were tested on a battery of tests, which examined reading proficiency (accuracy, speed and passage comprehension) and some control measures assessing language 
abilities. Italian tasks were presented to both groups of participants, while English tasks were only presented to bilinguals.

\subsubsection{Italian Tasks}

The Prove MT-3-Clinica: La valutazione delle abilità di Lettura e Comprensione per la scuola primaria e secondaria di I grado [27] evaluates the reading skills of children aged between 6 and 14 years. It is a standardized test that assesses reading rate (i.e., velocity, measured in syllables/seconds), accuracy (i.e., number of errors), and comprehension with two separate passages. Children are asked to read a passage aloud to assess velocity and accuracy. Reading comprehension instead consists of the silent reading of another passage, followed by a multiple-choice questionnaire about the story. Children are allowed to go back to the passage whenever they need to individuate the correct answer. Both passages differ according to the grade the child is attending.

The Test Neuropsicologico Lessicale per l'età evolutiva (TNL; [28]) is designed to measure expressive vocabulary in children between 3 and 9 years of age (although it should be noted that it was administered to older subjects as well). The naming task, which is part of the expressive vocabulary subtest, was administered to participants. Children are presented with 50 panels (A4 size) representing pictures they have to name aloud. The test examines the nouns category only. Nouns range from low to high frequency and are randomly ordered. Scores range between 0 and 50 .

The LITMUS SRep task-Italian version [29] (Italian adaptation by Levorato-not published) is a non-standardized school-age sentence repetition task that evaluates grammatical competence in Italian. This task has been developed within the European project Cost Action IS0804 'Language Impairment in a Multilingual Society', whose aim was to create a range of parallel tasks across a large number of languages to provide comparable results when assessing two or more languages. The task consists of 48 sentences pre-recorded by native Italian speakers and incorporated into a PowerPoint presentation for administration. The sentences include simple and complex syntactic structures, both language-independent (complex across languages), and language-specific (complex in the specific language) and are presented with increasing difficulty. The child listens to the sentences via headphones and then has to recall and repeat them aloud. Children are explicitly invited to repeat what they heard precisely. The 0-1 score scheme is used: a score of 1 is allocated if the sentence was repeated entirely verbatim, while a score of 0 if the sentence was repeated with one or more changes. Scores range between 0 and 48 .

\subsubsection{English Tasks}

The York Assessment of Reading Comprehension Passage Reading (YARC; [30]) evaluates the reading skills of children between 5 and 11 years. It measures reading rate (measured in seconds), accuracy and comprehension. Unlike the Italian reading task, this standardized test assesses reading decoding and comprehension in the same passage. Firstly, children are asked to read the passage aloud to assess the reading rate and accuracy; secondly, they have to answer the examiner's open-ended comprehension questions orally. Participants are invited to read the passage once more silently before starting comprehension questions, but they can also go back to the passage if needed. The test provides passages with seven levels of reading complexity (ranging from Beginners to Level 6); the right passage for each child is determined based on the performance on a word reading task, which is administered before the passage: the higher the number of words read correctly, the higher the reading level.

Word Finding Vocabulary Test: The Renfrew Language Scales [31] assesses expressive vocabulary in children aged between 3 and 8 years (although it should be noted that it was administered to older subjects as well), with possible scores between 0 and 50 . Children are shown 50 panels on a screen, representing pictures they have to name aloud, one at a time. Pictures represent both low and high-frequency nouns and are randomly ordered. 
The LITMUS SRep task-English version [32] evaluates grammatical competence in English. The task is structured similarly to the Italian version of the test, with pre-recorded sentences presented into a PowerPoint presentation to be repeated aloud by the child. The English sentence repetition task has 60 sentences targeting 15 syntactic structures of increasing complexity. The same 0-1 score scheme is used. Scores range between 0 and 60 .

\subsection{Procedure}

Data collection was started in April 2017 and finished in February 2020. Participants were tested individually on all tasks in a quiet room made available at school. One exception was the passage reading comprehension test, administered during school in small groups of two-to-three children, again in a quiet room during school hours. Children were outdistanced enough not to interfere with each other during the task. When it was not possible to administer the task collectively, it was administered individually. As mentioned above, bilinguals and monolinguals were tested at different times. Bilinguals were tested firstly. They were tested in Italian and English on separate days during the final part of the school year (springtime, between April and June). Monolinguals were only tested in Italian. Children from Italian monolingual schools in Grade 5 were tested during approximately the same period as bilingual children; Italian monolinguals mostly from Grade 1 and Grade 3 were tested at the beginning of the following school year (September). Thus, at the testing time, these monolingual first graders had just begun their second year of elementary school, and monolingual third graders had just started their fourth year. As shown above, this explains the significant age differences between the monolingual and bilingual groups in Grade 1 and Grade 3. This different testing period is taken into account in the statistical analysis.

Although a proficient English-speaking person administered English tests, English instructions and language items (e.g., sentences) were recorded by a native English speaker and presented as audio recordings through headphones to both groups of participants. English tasks were only presented to bilinguals.

The Ethic Committee of the University of Milano-Bicocca approved the study and parent's written consent was obtained for every child tested.

\subsection{Statistical Analysis}

To compare the performance on Italian tasks of the bilingual and the Italian monolingual groups in Grade 1, 3 and 5, an analysis of variance (GLM: General Linear Models) was performed, with group (bilingual and monolingual) and grade (first, third and fifth) as between subject factors and a covariate, testing time, calculated in terms of the number of days from the beginning of the year. Using the testing time as a covariate, the different ages between bilingual and monolingual first and third graders were controlled (bilingual and monolingual subgroups were tested at different times in the school years). Scores in a particular test were the dependent variable. Significant main effects and interactions are reported. Post hoc analyses corrected with Bonferroni were performed. An analysis of variance was also used to compare the performance of the three grades in the English tasks.

Correlations were used to verify whether the bilingual group's performance on Italian tasks was related to that on English tasks. We carried out correlation analyses to verify whether the bilinguals' performance in Italian was related to English performance. In these analyses, performances on each ability were correlated in the two languages. Since, in some cases, the two tasks in the two languages were not directly comparable because of raw scores from different tests, we used standard scores (as in the case of reading accuracy), calculated based on monolingual norms provided by the test, or the percentage scores of accuracy (as in the case of vocabulary, sentence repetition and reading comprehension). Reading speed was not correlated because it was measured differently in the two languages. As the York test for reading assessment does not provide norms in the case of Beginner-level reading, we excluded first-grader bilinguals from the correlational analysis of reading, as a significant portion of them were tested with the Beginner-level text. 


\section{Results}

The reading comprehension scores of nine bilingual first graders were missing because of the limited time available at the end of the school year; thus, it was impossible to administer the task individually or collectively. Missing data were replaced with the average score of the bilingual first-grade target group.

\subsection{Comparison between Bilinguals and Monolinguals on the Italian Tasks}

To answer the first research question, whether an Italian-English simultaneous immersive education impacts the acquisition of Italian (L1) and the literacy development in Italian, we examined the Italian performance of the bilingual group compared to that of the monolinguals.

First, we analyzed measures assessing oral language abilities. As is evident in Figure 1, expressive vocabulary was found to improve across the three grades, both in monolingual and bilingual children.

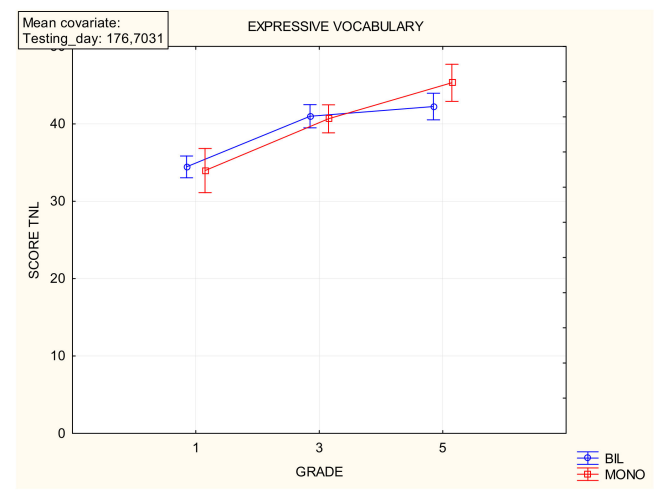

Figure 1. Raw scores $(\mathrm{Max}=50)$ at Italian expressive vocabulary (TNL) in Grade 1, 3 and 5 of monolingual and bilingual children. Bars indicate a confidence interval at 0.95 .

GLM analysis on raw scores from the Test Neuropsicologico Lessicale per l'età evolutiva (TNL) with group (monolingual and bilingual) and grades $(1,3,5)$ as between subject factors, and testing time as a covariate confirmed this observation: only an effect of grade was found $F(2,185)=40.5, \eta^{2}=0.30, p<0.001$ (in our analyses, we report the partial Eta-square $\left(\eta^{2}\right)$, which is a measure of effect size). Bonferroni-corrected post hoc results confirmed that all three grades differed from each other $(p<0.05)$. No significant group difference was found.

As shown in Figure 2, performances in the sentence repetition task improved from first to third Grade in both groups of children. In third Grade, we observe a ceiling effect (total number of responses $=48$ ).

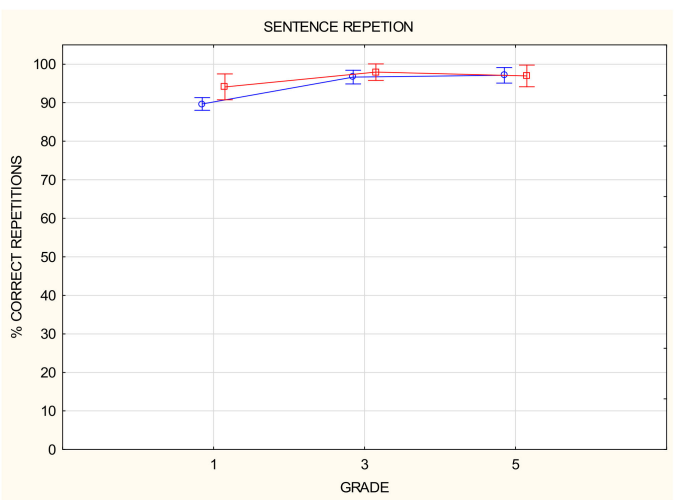

Figure 2. Scores $(\max =48)$ at the Italian sentence repetition task in Grade 1, 3 and 5 of monolingual and bilingual children. Bars indicate a confidence interval at 0.95 . 
As expected, a GLM analysis on scores from the LITMUS SRep task-Italian version with group and grades as between subject factors and testing time as a covariate only found an effect of grade $\mathrm{F}(2185)=14.6, \eta^{2}=0.14, p<0.005$. In addition, Bonferroni-corrected post hoc results showed that first graders differed from third and fifth graders $(p<0.001)$.

To summarize, GLM analyses revealed an effect of grade on language proficiency, indicating positive gains in both groups at lexical and morpho-syntactic levels.

Then, we moved on to analyze passage reading proficiency measures. As shown in Figure 3, reading speed, which in Prove MT-3-Clinica is measured in syllables/seconds, similarly increased across grades in the two groups.

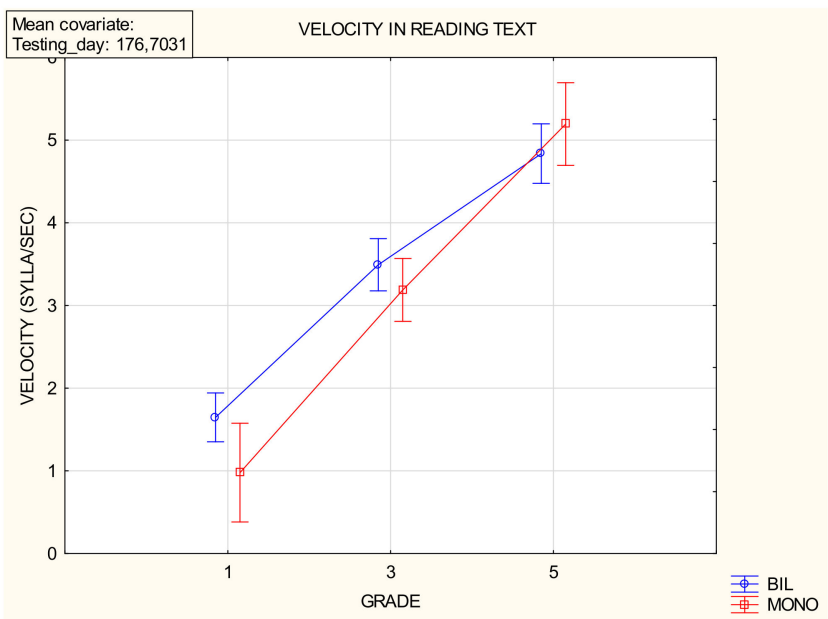

Figure 3. The reading velocity (syllable/seconds) of a text (MT) in Italian in Grade 1, 3 and 5 of monolingual and bilingual children. Bars indicate a confidence interval at 0.95 .

Statistical analysis confirmed this observation: an effect of grade was found $\mathrm{F}(2185)=121.3$, $\eta^{2}=0.56, p<0.001$. Bonferroni-corrected post hoc results revealed that all three grades differed from each other $(p<0.001)$. Furthermore, the testing time was significant $\mathrm{F}(1185)=16.43$, $\eta^{2}=0.08, p<0.001$, although this effect was low, as indicated by the $\eta^{2}$. No significant difference was found in the performance of monolingual and bilingual groups.

In Figure 4, we can observe scores in reading accuracy expressed in terms of the number of errors. As can be seen, bilingual children made slightly more errors than monolinguals, especially in Grade 1. Additionally, there was a definite improvement in performance from Grade 1 to Grade 3 in both groups.

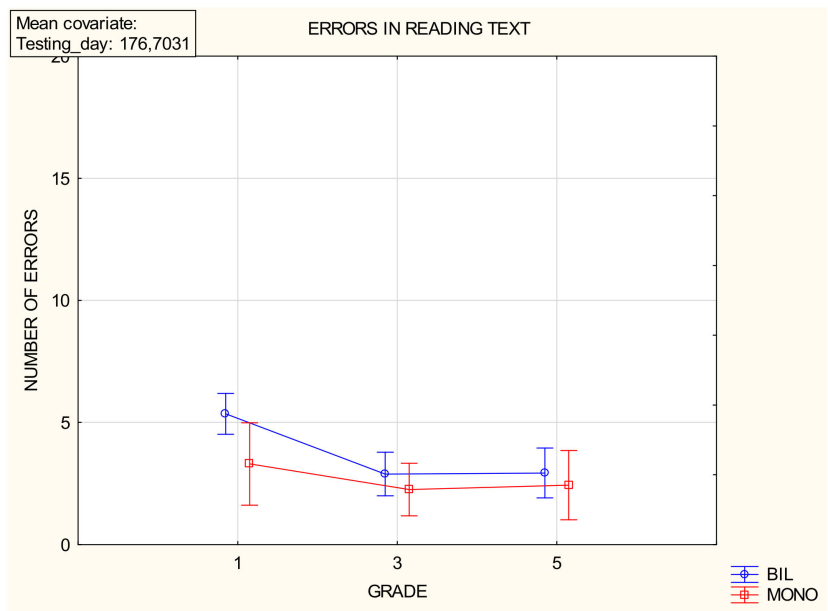

Figure 4. Errors at reading a text (MT) in Italian in Grade 1, 3 and 5 of monolingual and bilingual children. Bars indicate a confidence interval at 0.95 . 
The statistical analysis only revealed an effect of grade $F(2185)=6.06, \eta^{2}=0.06$, $p<0.005$. Bonferroni-corrected post hoc results showed that first graders differed from third and fifth graders $(p<0.01)$. Differences in the performance of monolingual and bilingual groups did not turn out to be significant.

Finally, we analyzed passage reading comprehension. Figure 5 illustrates the performance of the participants.

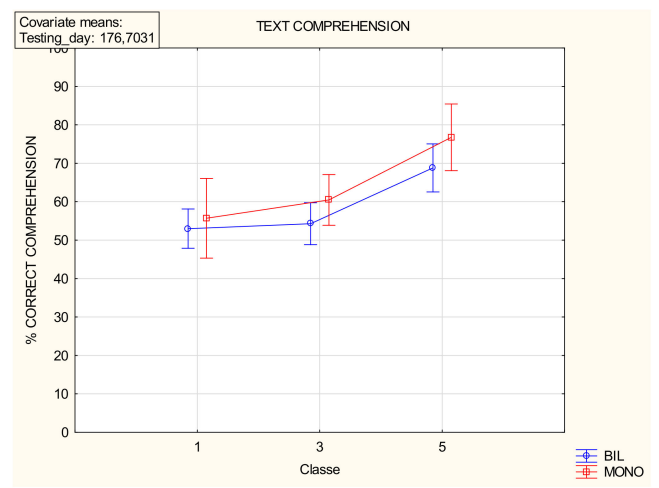

Figure 5. Raw scores at the reading comprehension (MT) in Italian in Grade 1, 3 and 5 of monolingual and bilingual children. Bars indicate a confidence interval at 0.95 .

The statistical analysis revealed an effect of grade $\mathrm{F}(2185)=11.36, \eta^{2}=0.10, p<0.001$. Bonferroni-corrected post hoc results showed that first and third graders differed from fifth graders $(p<0.001)$.

In conclusion, robust effects of grade were found in all the Italian tasks evaluated. When controlling for the time of testing, which was different for monolingual children in Grades 1 and 3, we did not find group differences in any measure.

\subsection{English Language and Reading Development within the Bilingual Group}

To observe the impact of an Italian-English simultaneous immersion program on the development of language and literacy skills in English (L2), we examined the English performance of the bilingual group across Grade 1, 3 and 5 .

First, we analyzed English expressive vocabulary and the sentence repetition task in English. Only 23\% of bilingual children had English vocabulary scores within the monolingual normal limits, but this is a generally common bilingual feature (e.g., Bialystok, Luk, Peets and Yang, 2010; Hoff, Rumiche, Burridge, Ribot and Welsh, 2014). We must point out that in Grade 5, standard scores were just indicative, as the test is standardized for children up to 8.5 years old.

As shown in Figures 6 and 7, both skills were found to improve across grades, although this was to a greater extent from Grade 1 to 3 .

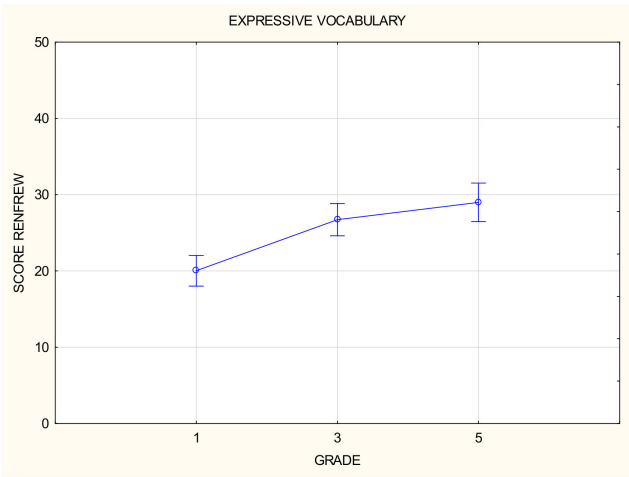

Figure 6. Raw scores (Max = 50) in English expressive vocabulary (Renfrew) in Grade 1, 3 and 5 of bilingual children. Bars indicate a confidence interval at 0.95 . 


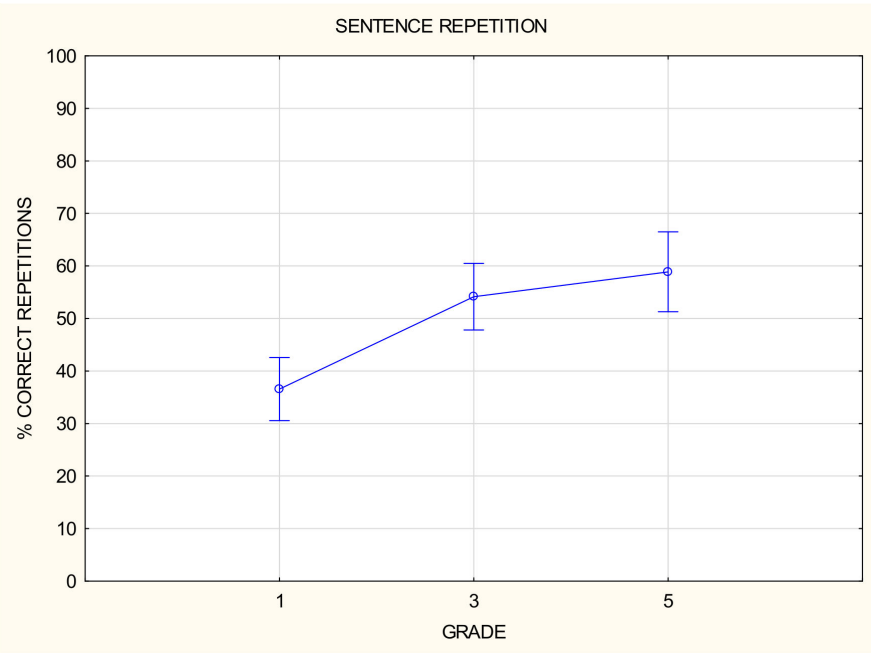

Figure 7. Scores $(\mathrm{Max}=60)$ in the English sentence repetition task in Grade 1, 3 and 5 of bilingual children. Bars indicate a confidence interval at 0.95 .

GLM analysis on raw scores in the Word Finding Vocabulary Test with grades $(1,3$ and 5 ) as between subject factor showed an effect of grade $\mathrm{F}(2118)=18.2, \eta^{2}=0.24, p<0.001$. The Bonferroni-corrected post hoc test confirmed that bilingual first graders differed from third and fifth graders $(p<0.001)$. An effect of grade was also found in the LITMUS SRep task -English version F (2118) $=13, \eta^{2}=0.18, p<0.001$. Bonferroni-corrected post hoc results showed that first graders differed from third and fifth graders $(p<0.001)$.

Secondly, we analyzed reading speed and accuracy through the York Assessment of Reading Comprehension Passage Reading (YARC). In Figures 8 and 9, we can observe a similar trend across grades for both measures. Unlike the Italian MT-3 test for reading (which measures velocity in syllables/seconds), YARC reading speed is the total time in seconds that the child takes to read the passage.

Bilingual children in Grade 3 took more time to read the passage than bilingual first graders, but the performance of bilingual children definitely improved in Grade 5 .

Regarding accuracy, third graders made more errors while reading a text (accuracy score is an error score) compared to both first and fifth graders.

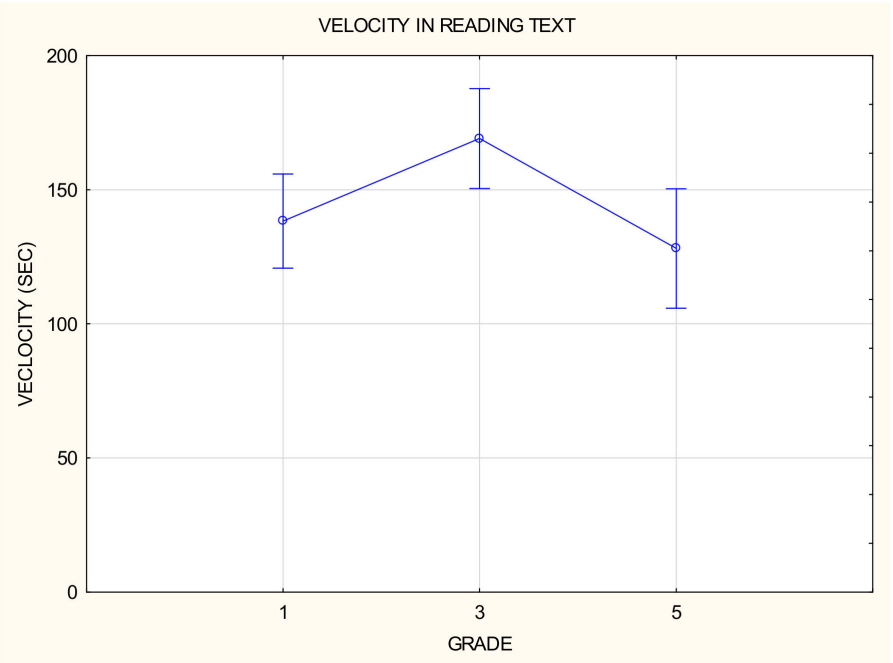

Figure 8. Reading velocity of a text (York) in English in Grade 1, 3 and 5 of bilingual children. Bars indicate a confidence interval at 0.95 . 


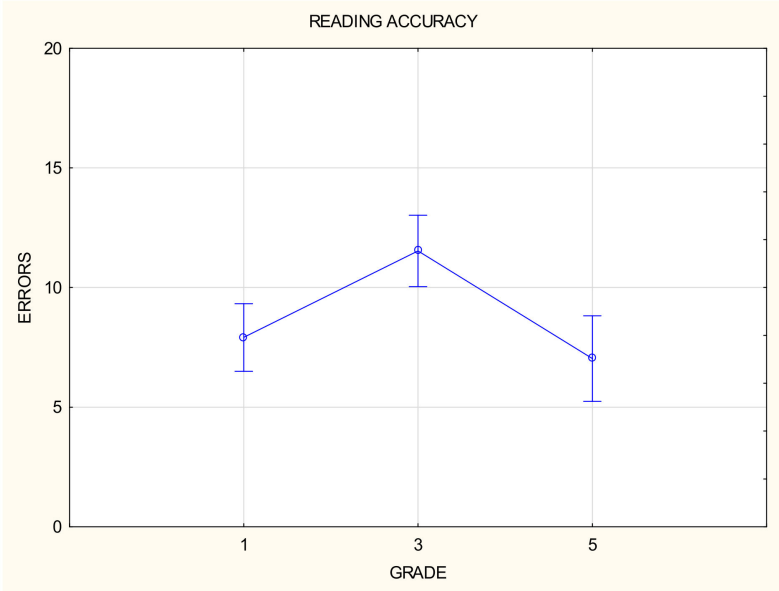

Figure 9. Errors in reading an English text (York) in Grade 1, 3 and 5 of bilingual children. Bars indicate a confidence interval at 0.95 .

Statistical analyses found an effect of grade both in reading rate, $\mathrm{F}(2118)=4.66$, $\eta^{2}=0.07, p<0.05$, and in reading accuracy, $\mathrm{F}(2118)=0.918, \eta^{2}=0.13, p<0.001$. Bonferronicorrected post hoc results revealed that third graders differed from fifth graders $(p<0.05)$ in reading rate, with fifth graders outperforming third graders; in reading accuracy, Bonferroni-corrected post hoc results showed that first graders differed from third graders $(p<0.005)$, with first graders being more accurate than third graders (fewer errors). Third graders differed from fifth graders $(p<0.001)$, with third graders being less accurate (more errors) than fifth graders. A total of $82 \%$ of the children were more than 1.50 standard deviations below the mean on reading velocity, and $97 \%$ were more than 1.50 standard deviations below the mean on reading accuracy, calculated based on English monolingual norms.

Reading comprehension performance is displayed in Figure 10. As can be seen, there is an improvement from Grade 3 to 5, although the difference was not statistically significant. We could not estimate standard scores for reading comprehension as the test provides norms based on two passages, while we only administered one.

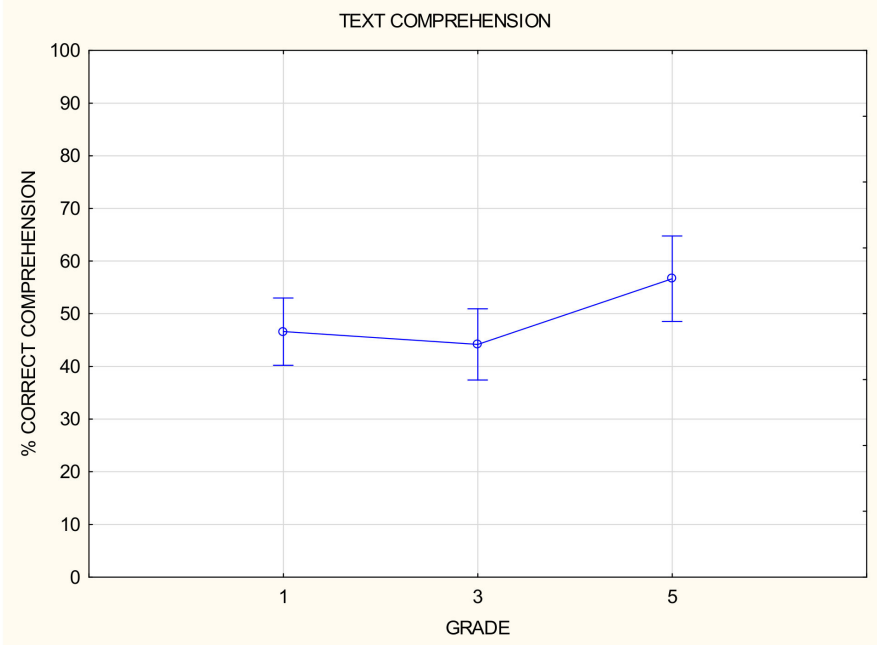

Figure 10. English reading comprehension (York) scores in Grade 1, 3 and 5 of bilingual children. Bars indicate a confidence interval at 0.95 .

In conclusion, the effect of grade was found in all of the English measures in the ItalianEnglish bilingual group, with the only exception being reading comprehension. However, regarding English reading proficiency measures (i.e., reading rate and accuracy), a weaker 
performance was revealed in Grade 3 children. Still, there was a final improvement in Grade 5 by the end of primary school.

\subsection{Correlations between Italian and English Skills in the Bilingual Group}

As far as language measures are concerned, a significant positive correlation was found between expressive vocabulary measures in Italian and English, $\mathrm{r}=0.47, p<0.001$. A significant positive correlation was also found between the scores in the LITMUS SRep tasks (Italian and English version) $r=0.40, p<0.001$.

Reading measures - involving only third and fifth graders - revealed that reading errors also correlated $\mathrm{r}=0.37, p<0.05$ : those who made more errors in Italian also did so in English. Reading comprehension in the two languages correlated, $\mathrm{r}=0.24, p=0.03$. We must point out the difference between the Italian and English reading comprehension tasks. While in Italian, the responses to the questions were written, and different texts were used to test technical reading and reading comprehension, in English, they were administered orally, and the same passage was used to test both technical reading and reading comprehension.

\subsection{Correlations among the Various Abilities in the Monolingual and Bilingual Groups}

To gain further information about the development of language and literacy in Italian, we performed several correlation analyses among the various abilities in the two groups separately.

We first analyzed the correlations within the monolingual group. As evident in the correlation matrix in Table 2, vocabulary and reading speed correlated with most of the other variables. Specifically, vocabulary correlated with reading and sentence repetition. Reading speed correlated with other reading measures and sentence repetition.

Table 2. The correlation matrix of the Italian measures within the monolingual group. Only significant correlations are marked $(p<0.05)$.

\begin{tabular}{cccccc}
\hline Measure & Vocab. & Read Speed & Read Acc. & Read Compr. & Sent. Rep. \\
\hline Vocab. & & 0.56 & & 0.43 & 0.56 \\
Read speed & 0.56 & & -0.30 & 0.34 & 0.30 \\
Read acc. & & -0.30 & & & \\
Read compr. & 0.43 & 0.35 & & & \\
Sent. rep. & 0.56 & 0.29 & & & \\
\hline
\end{tabular}

The same picture emerges for the bilingual group, as shown in Table 3

Table 3. The correlation matrix of the Italian measures within the bilingual group. Only significant correlations are marked $(p<0.05)$.

\begin{tabular}{cccccc}
\hline Measure & Vocab. & Read Speed & Read Acc. & Read Compr. & Sent. Rep. \\
\hline Vocab. & & 0.55 & -0.27 & 0.30 & 0.46 \\
Read speed & 0.55 & & -0.54 & 0.35 & 0.41 \\
Read acc. & -0.27 & -0.54 & & -0.24 & -0.20 \\
Read compr. & 0.29 & 0.35 & -0.24 & & 0.22 \\
Sent. rep. & 0.46 & 0.41 & -0.20 & -0.22 & \\
\hline
\end{tabular}

In summary, these correlations, which are similar for the mono- and bilingual children, show that various reading and language (vocabulary and sentence repetition) measures develop similarly in both groups across the two languages.

Finally, Table 4 reports the correlations among the various abilities in English in the bilingual group. We found multiple correlations between vocabulary, reading speed and comprehension and sentence repetition. Some reading measures correlated with other reading measures. 
Table 4. The correlation matrix of the English measures within the bilingual group. Only significant correlations are marked in red $(p<0.05)$.

\begin{tabular}{cccccc}
\hline Measure & Vocab. & Read Speed & Read Acc. & Read Compr. & Sent. Rep. \\
\hline $\begin{array}{c}\text { Vocab. } \\
\text { Read speed }\end{array}$ & 0.21 & 0.21 & & 0.24 & 0.61 \\
$\begin{array}{c}\text { Read acc. } \\
\text { Read compr. }\end{array}$ & 0.24 & 0.44 & 0.44 & & -0.19 \\
$\quad$ Sent. rep. & 0.61 & 0.24 & -0.19 & & \\
\hline
\end{tabular}

\section{Discussion}

The study aimed to investigate the effects of bilingual education on the literacy attainment of primary school Italian-English bilinguals. Concerns and queries surround the benefits of bilingual education on literacy success and raise the question of whether learning to read in Italian (L1) is delayed when simultaneously learning to read in English (L2). Thus, we were primarily interested in verifying whether the Italian reading performance of early bilingual primary school children differed significantly from that exhibited by their Italian monolingual peers. To do so, the Italian language and reading performance of three groups of bilinguals educated in 50:50 Italian-English immersion programs (with Italian as their L1 and majority language) in Grades 1, 3 and 5 were compared to that of Italian monolingual peers living in Italy. Secondly, we examined whether there were improvements in English language and reading across the three grades in the bilingual group. Therefore, we compared the performance of the three grades. Thirdly, we were interested in verifying whether the bilinguals' language and reading attainment in Italian were related to their English performance. We performed correlations between the same skills evaluated in the two languages in the bilingual group. Fourthly, we aimed to verify the hypothesis that competence in oral language supports literacy development. We tested this hypothesis by correlating language and reading measures both in Italian and English. In the rest of the discussion, we consider each aspect.

\subsection{Learning to Read Two Languages Simultaneously}

Regarding whether Italian literacy is influenced by learning to read in two languages simultaneously, we predicted that there should not have been significant differences between the monolinguals' and bilinguals' competence in Italian. In line with this prediction, we found development across grades in language and literacy skills in both groups, which mirrors a consolidation in language and reading skills. On all language and reading measures, the two groups did not differ. This finding agrees with Costa et al. [33], who measured decoding skills through reading lists of words and pseudowords. These authors found that the monolingual and bilingual groups did not differ in any measures of reading. In our study, we measured reading skills through a text. This choice meant that not only decoding was assessed, but also other skills, such as vocabulary knowledge, morpho-syntactic competence and the ability to make inferences. In our sample, bilinguals did not differ from monolinguals in Italian reading, even in first and third Grade.

We found an improvement across grades in all the tasks considered. However, we should note that in some cases, the improvement was significant only from Grade 1 to Grade 3 (that was the case for sentence repetition task and reading accuracy) or from Grade 3 to Grade 5 (reading comprehension). This could be attributed to the cross-sectional nature of the study.

In summary, bilingual and monolingual children performed similarly in Italian, in line with the literature. Thus, we can conclude that learning to read in Italian and English simultaneously does not affect reading performance in Italian - when bilingual exposure occurs by three years of age. 


\subsection{Development in English Language and Literacy}

As regards the impact of an Italian-English immersion program on the development of language and literacy skills in English (L2), we predicted that there should be an improvement across the three grades.

Considering English language measures, that is, expressive vocabulary and morphosyntactic abilities, we found an improvement from Grade 1 to Grade 3, but the difference between Grade 3 and Grade 5 was not found to be statistically significant. However, this may be due to the cross-sectional nature of our study.

Looking at English reading proficiency, bilinguals were shown to improve their reading velocity by Grade 5, even if performance in Grade 3 was slower. We observed the same trend for reading accuracy, as third graders were less accurate than first graders, but the performance improved by Grade 5 . Moreover, almost all children achieved performance within English monolingual norms. As regards English reading comprehension, the performance across grades did not show a significant improvement.

In conclusion, the downfalls we observed in the bilingual group could plausibly be attributed to the cross-sectional design of the present study.

\subsection{Language and Literacy Development in Italian and English}

The third aim of the study was to verify whether the language and reading attainments exhibited by the bilingual children in Italian were related to their English performance. Significant correlations were found between the Italian and the English performances on various measures. This indicates a similar performance in the two languages and a similar development across grades. If we overview these findings, we can conclude that the significant correlations between Italian and English scores on various measures indicate that proficiency in one language is mirrored by competence in another language. These correlations also suggest that certain language and reading skills might be transferred from one language to the other. Plausibly, this cross-linguistic transfer is more likely to occur from Italian to English because Italian is the majority language and has a shallow orthography and a less complex phonological structure compared to English.

\subsection{The Relation between Language and Reading}

As discussed above, it is well established that oral language competence is a predictor of reading outcomes. Thus, another aim of the study was to verify whether oral language abilities were related to reading performance. Considering Italian, we found that vocabulary was related with most of the reading measures in the monolingual group and all of the reading measures in the bilinguals, as expected [34]. Morpho-syntax, as measured by sentence repetition, was also found to relate with reading measures in the bilingual group. This result was in line with Kovelmann et al. [3] and Prevoo et al. [35], who found strong associations between language proficiency and reading in bilingual children. In the monolingual group, the morpho-syntactic measure was only related with reading speed among the reading measures.

Looking at the bilinguals' English performance, vocabulary was shown to be related to most of the reading measures, and the morpho-syntactic measure was related to some of the reading measures.

Thus, it stands to reason that proficiency in the language of school instruction is a necessity for understanding the teacher and a forerunner to literacy attainment [35].

In conclusion, our results indicate that acquiring literacy simultaneously in two languages does not negatively affect literacy in the native language. Educational policy should take this into account and promote early literacy and early exposure to an L2 oral language. This study involved children exposed to English by age 3; a possible extension is to investigate a bilingual educational system, where English is learned later in both oral and written form. A further development would be to carry out a longitudinal study, although this may be practically very hard to do, as it requires the collection of data over 5 years. 


\section{Conclusions}

Our hypotheses were all confirmed by the results of the present study. To summarize, we found no evidence that a bilingual learning environment hinders the L1 language and reading proficiency in a simultaneous Italian-English immersion program when exposure to English L2 occurs from the age of 3. We found bilingual students improved in language and reading both in Italian and English. Nevertheless, in some English reading skills (velocity and accuracy), we found a downfall in Grade 3 performance, but this could be attributed to the study's sampling. We found positive correlations between the bilinguals' Italian and English performances, conjecturing a positive transfer of skills from one language to another, presumably from Italian to English, which could support the same ability in English, the second language. Language abilities in one language were found to correlate with reading abilities in the same language, supporting the idea that oral language supports reading development. All in all, speaking and reading more than one language does not appear to be detrimental, even when learning to read has just started.

Author Contributions: Conceptualization, F.C. and M.T.G.; methodology, F.C. and M.T.G.; investigation, F.C.; data curation, F.C.; data analysis, M.T.G.; writing-original draft preparation, F.C.; writing-review and editing, F.C. and M.T.G. All authors have read and agreed to the published version of the manuscript.

Funding: The first author was funded by a scholarship supporting her PhD studies from the University of Milano-Bicocca.

Institutional Review Board Statement: The study was conducted according to the guidelines of the Declaration of Helsinki, and approved by the Institutional Review Board (or Ethics Committee) of the University of Milano-Bicocca (protocol code 280 and date of approval 2 March 2017).

Informed Consent Statement: Informed consent was obtained from all subjects involved in the study.

Data Availability Statement: Data can be obtained by the corresponding author.

Conflicts of Interest: The authors declare no conflict of interest.

\section{References}

1. Diamond, J.M. The World until Yesterday: What Can We Learn from Traditional Societies? Penguin: London, UK, 2013.

2. Baker, C. Foundations of Bilingual Education and Bilingualism; Multilingual Matters: Bristol, UK, 2011.

3. Kovelman, I.; Baker, S.A.; Petitto, L.A. Age of first bilingual language exposure as a new window into bilingual reading development. Biling. Lang. Cogn. 2008, 11, 203-223. [CrossRef]

4. Slavin, R.E.; Cheung, A. A synthesis of research on language of reading instruction for English language learners. Rev. Educ. Res. 2005, 75, 247-284. [CrossRef]

5. Cummins, J. Interdependence of first-and second-language proficiency in bilingual children. Lang. Process. Biling. Child. 1991, 70, 89. [CrossRef]

6. Cummins, J. The role of primary language development in promoting educational success for language minority students. In Schooling and Language Minority Students. A Theoretical Framework; California State Department of Education: Sacramento, CA, USA, 1981; pp. 3-49.

7. Berens, M.S.; Kovelman, I.; Petitto, L.A. Should bilingual children learn reading in two languages at the same time or in sequence? Biling Res. J. 2013, 36, 35-60. [CrossRef] [PubMed]

8. Christian, D.; Howard, E.R.; Loeb, M.I. Bilingualism for all: Two-way immersion education in the United States. Theory Into Pract. 2000, 39, 258-266. [CrossRef]

9. Genesee, F.; Jared, D. Literacy development in early French immersion programs. Canadian Psychology/Psychologie canadienne 2008, 49, 140. [CrossRef]

10. Lindholm-Leary, K.; Hernández, A. Achievement and language proficiency of Latino students in dual language programmes: Native English speakers, fluent English/previous ELLs, and current ELLs. J. Multiling. Multicult. Dev. 2011, 32, 531-545. [CrossRef]

11. Turnbull, M.; Lapkin, S.; Hart, D. Grade 3 immersion students' performance in literacy and mathematics: Province-wide results from Ontario (1998-1999). Can. Mod. Lang. Rev. 2001, 58, 9-26. [CrossRef]

12. Abu-Rabia, S.; Siegel, L.S. Reading, syntactic, orthographic, and working memory skills of bilingual Arabic-English speaking Canadian children. J. Psycholinguist Res. 2002, 31, 661-678. [CrossRef]

13. Collier, V.P. A synthesis of studies examining long-term language minority student data on academic achievement. Biling. Res. J. 1992, 16, 187-212. [CrossRef] 
14. López, M.G.; Tashakkori, A. Effects of a two-way bilingual program on the literacy development of students in kindergarten and first grade. Biling. Res. J. 2004, 28, 19-34. [CrossRef]

15. Oller, D.K.; Eilers, R.E. Language and Literacy in Bilingual Children; Multilingual Matters: Bristol, UK, 2002; Volume 2. [CrossRef]

16. Rolstad, K.; Mahoney, K.; Glass, G.V. The big picture: A meta-analysis of program effectiveness research on English language learners. Educ. Policy 2005, 19, 572-594. [CrossRef]

17. Thomas, W.; Collier, V. The multiple benefits of dual language. Educ. Leadersh. 2003, 61, 61.

18. Bialystok, E. Bilingual education for young children: Review of the effects and consequences. Int. J. Biling. Educ. Biling. 2018, 21, 666-679. [CrossRef]

19. Halle, T.; Hair, E.; Wandner, L.; McNamara, M.; Chien, N. Predictors and outcomes of early versus later English language proficiency among English language learners. Early Child. Res. Q. 2012, 27, 1-20. [CrossRef] [PubMed]

20. Flege, J.E.; Yeni-Komshian, G.H.; Liu, S. Age constraints on second-language acquisition. J. Mem. Lang. 1999, 41, 78-104. [CrossRef]

21. Perani, D.; Abutalebi, J.; Paulesu, E.; Brambati, S.; Scifo, P.; Cappa, S.F.; Fazio, F. The role of age of acquisition and language usage in early, high-proficient bilinguals: An fMRI study during verbal fluency. Hum. Brain Mapp. 2003, 19, 170-182. [CrossRef]

22. Bialystok, E.; Senman, L. Executive processes in appearance-reality tasks: The role of inhibition of attention and symbolic representation. Child Dev. 2004, 75, 562-579. [CrossRef]

23. Bialystok, E.; Shapero, D. Ambiguous benefits: The effect of bilingualism on reversing ambiguous figures. Dev. Sci. 2005, 8, 595-604. [CrossRef]

24. Bialystok, E. Factors in the growth of linguistic awareness. Child Dev. 1986, 57, 498-510. [CrossRef]

25. Paradis, J.; Genesee, F.; Crago, M.B. Dual Language Development and Disorders. In A Handbook on Bilingualism and Second Language Learning, 3rd ed.; Brookes Publishing: Baltimore, MD, USA, 2011.

26. Bonifacci, P.; Tobia, V. Crossing barriers: Profiles of reading and comprehension skills in early and late bilinguals, poor comprehenders, reading impaired, and typically developing children. Learn. Individ. Differ. 2016, 47, 17-26. [CrossRef]

27. Cornoldi, C.; Carretti, B. Prove MT-3 Clinica: La Valutazione Delle Abilità di Lettura e Comprensione per La Scuola Primaria e Secondaria di I Grado; Giunti Editore: Florence, Italy, 2016.

28. Cossu, G. TNL-Test Neuropsicologico Lessicale per l'età Evolutiva [Lexical Neuropsychological Test for Children]; Hogrefe: Newburyport, MA, USA, 2013.

29. Marinis, T.; Armon-Lotem, S. Sentence Repetition; Assessing Multilingual Children: Disentangling Bilingualism from Language Impairment; Multilingual Matters: Bristol, UK, 2015; pp. 95-124.

30. Snowling, M.J.; Stothard, S.E.; Clarke, P.; Bowyer-Crane, C.; Harrington, A.; Truelove, E.; Hulme, C. YARC York Assessment of Reading for Comprehension Passage Reading; GL Publishers: Guwahati, India, 2009.

31. Renfrew, C.; Mitchell, P. Word finding vocabulary test: The Renfrew language scales. Speechmark Publishing Ltd. Rhoades, BL, Greenberg, MT, Lanza, ST, \& Blair, C. (2011). Demographic and familial predictors of early executive function development: Contribution of a person-centered perspective. J. Exp. Child Psychol. 1997, 108, 638-662.

32. Marinis, T.; Chiat, S.; Armon-Lotem, S.; Gibbons, D.; Gipps, E. School-Age Sentence Imitation Test (SASIT); University of Reading: Reading, UK, 2010.

33. Costa, F.; Guasti, M.T.; Sharley, S. Double Literacy Effects on Language and Reading Skills in Italian-English Primary School Children. J. Monolingual Biling. Speech 2020, 2, 185-218. [CrossRef]

34. Duff, F.J.; Reen, G.; Plunkett, K.; Nation, K. Do infant vocabulary skills predict school-age language and literacy outcomes? J. Child Psychol. Psychiatry 2015, 56, 848-856. [CrossRef]

35. Prevoo, M.J.L.; Malda, M.; Mesman, J.; van IJzendoorn, M.H. Within-and cross-language relations between oral language proficiency and school outcomes in bilingual children with an immigrant background: A meta-analytical study. Rev. Educ. Res. 2016, 86, 237-276. [CrossRef] 